\title{
$k$-PARTITIONS AND A CHARACTERIZATION FOR COMPACT UNIONS OF $k$ STARSHAPED SETS
}

\author{
MARILYN BREEN
}

(Communicated by William J. Davis)

\begin{abstract}
For natural numbers $k$ and $j$, define $\sigma(1, j)=j+2, \sigma(2,1)=6$, and $\sigma(k, j)=k^{2} j+1$ otherwise. The set $S$ in $R^{d}$ has property $A(k)$ if and only if $S$ is a finite union of one-dimensional convex sets and for every $\sigma(k, 1)$-member subset $F$ of $S$ there correspond points $c_{1}, \ldots, c_{k}$ (depending on $F$ ) such that each point of $F$ sees via $S$ some $c_{i}, 1 \leqslant i \leqslant k$. The following results are established.

(1) Let $k$ and $j$ be fixed natural numbers, and let $\mathscr{L}$ be a collection of sets such that every $j+1$ members meet in at most one point. Then $\mathscr{L}$ has a $k$-partition $\mathscr{L}_{1}, \ldots, \mathscr{L}_{k}$ with $\bigcap\left\{L: L\right.$ in $\left.\mathscr{L}_{i}\right\} \neq \varnothing$ if and only if every $\sigma(k, j)$ or fewer members of $\mathscr{L}$ have such a $k$-partition.

(2) Let $S$ be compact in $R^{d}$. The set $S$ is a union of $k$ starshaped sets if and only if there is a sequence of compact sets $\left\{S_{i}\right\}$ converging to $S$ (relative to the Hausdorff metric) such that each set $S_{i}$ satisfies property $A(k)$.

The first result yields a piercing number for certain families of sets, while the second provides a characterization for compact unions of $k$ starshaped sets in $R^{d}$.
\end{abstract}

1. Introduction. We begin with some definitions. Let $S$ be a set in $R^{d}$. For points $x$ and $y$ in $S$, we say $x$ sees $y$ via $S$ if and only if the segment $[x, y]$ lies in $S$. The set $S$ is starshaped if and only if there is some point $p$ in $S$ such that $p$ sees via $S$ each point of $S$. The set of all such points $p$ is the (convex) kernel of $S$, denoted $\operatorname{ker} S$. Finally, a family $\mathscr{L}$ of sets is said to have a $k$-partition (equivalently, $\mathscr{L}$ is called $k$-pierceable) if and only if $\mathscr{L}$ may be partitioned into $k$ subfamilies, each having a nonempty intersection.

A well-known theorem of Krasnosel'skii [7] states that if $S$ is a nonempty compact set in $R^{d}, S$ is starshaped if and only if every $d+1$ points of $S$ see via $S$ a common point. Some Krasnosel'skii-type theorems for unions of starshaped sets have been obtained in $[3,1]$. Each of these requires that certain conditions be satisfied by finite subsets of set $S$, and by [2, Example 4], no finite Krasnosel'skii number exists to characterize arbitrary unions of two or more compact starshaped sets, even in the plane.

However, finite Krasnosel'skii numbers do exist for certain kinds of sets. In particular, by [4, Corollary 2], for $S$ a finite union of one-dimensional convex sets in $R^{d}, S$ is a union of two starshaped sets if and only if for every 6-point subset $T$ of $S$

Received by the editors September 22, 1986.

1980 Mathematics Subject Classification (1985 Revision). Primary 52A30, 52A35.

Key words and phrases. K-partitions, unions of starshaped sets, Krasnosel'skii-type theorems. 
there correspond points $p_{1}$ and $p_{2}$ (depending on $T$ ) such that each point of $T$ sees via $S$ either $p_{1}$ or $p_{2}$. Here we generalize the previous result to unions of $k$ starshaped sets and in the process obtain a piercing number for certain families of sets. As in [1], while not every compact union $G$ of $k$ starshaped sets will satisfy our hypothesis, $G$ will be the limit, relative to the Hausdorff metric, for a sequence whose terms do satisfy it. This yields a characterization for compact unions of $k$ starshaped sets in $R^{d}$.

The reader is referred to Valentine [10] and to Lay [8] for a discussion of starshaped sets, to Danzer, Grünbaum, and Klee $[5,6]$ for a discussion of $k$-partitions and $k$-pierceable families, and to Nadler [9] for information on the Hausdorff metric.

2. The results. The following definitions and easy lemma will be helpful.

Definition 1. For natural numbers $k$ and $j$, define $\sigma(1, j)=j+2, \sigma(2,1)=6$, and $\sigma(k, j)=k^{2} j+1$ otherwise.

Definition 2. Let $S \subseteq R^{d}$. We say that $S$ has property $A(k)$ if and only if $S$ is a finite union of one-dimensional convex sets and for every $\sigma(k, 1)$-member subset $F$ of $S$ there correspond points $c_{1}, \ldots, c_{k}$ (depending on $F$ ) such that each point of $F$ sees via $S$ some $c_{i}, 1 \leqslant i \leqslant k$.

LEMMA 1. For all natural numbers $k$ and $j, \sigma(k, j) \geqslant k^{2} j+1$. For $k \geqslant 2, \sigma(k, j)$ $\geqslant \sigma(k-1, j)+k j+1$.

Proof. The first statement is clear. To check the second statement, notice that if $k=2$ and $j=1$, then

$$
\sigma(2,1)=6=\sigma(1,1)+2+1 .
$$

If $k=2$ and $j \geqslant 2$, then

$$
\sigma(2, j)=4 j+1 \geqslant 3 j+3=\sigma(1, j)+2 j+1 .
$$

If $k \geqslant 3$ then

$$
\sigma(k, j)=k^{2} j+1>k^{2} j-j(k-1)+2=\sigma(k-1, j)+k j+1 .
$$

The first result extends [4, Theorem 2] and yields a $k$-piercing number for certain families of sets.

THEOREM 1. Let $k$ and $j$ be fixed natural numbers, and let $\mathscr{L}$ be a collection of sets such that every $j+1$ members meet in at most one point. Then $\mathscr{L}$ has a $k$-partition if and only if every $\sigma(k, j)$ or fewer members of $\mathscr{L}$ have a $k$-partition.

Proof. Clearly we need to prove only the sufficiency. Without loss of generality, assume that $\mathscr{L}$ has at least $\sigma(k, j)$ members. We proceed by induction on $k$. If $k=1$, then by hypothesis every $\sigma(1, j)=j+2$ members of $\mathscr{L}$ meet. Hence some $j+1$ of these members meet in a unique point $x$, every member of $\mathscr{L}$ necessarily contains $x$, and $\mathscr{L}$ has a 1 -partition.

Inductively, assume the result is true for natural numbers $k<n$ to prove for $n$, $n \geqslant 2$. Let $\mathscr{L}$ be a collection of sets satisfying our hypothesis, with every $\sigma(n, j)$ or fewer members of $\mathscr{L}$ having an $n$-partition. 
Let $\mathscr{L}^{\prime}$ denote a set consisting of exactly $\sigma(n, j) \geqslant n^{2} j+1$ members of $\mathscr{L}$. By hypothesis, $\mathscr{L}^{\prime}$ has an $n$-partition. Moreover, by the pigeon-hole principle, some $n j+1$ members of $\mathscr{L}^{\prime}$ belong to the same partition set and hence contain a common point, say $x$. Let $\mathscr{L}_{0}$ denote a set of exactly $n j+1$ members of $\mathscr{L}^{\prime}$ containing $x$.

Let $\mathscr{M}$ denote the members of $\mathscr{L}$ which fail to contain $x$, and assume $\mathscr{M} \neq \varnothing$. We assert that $\mathscr{M}$ has an $(n-1)$-partition. Let $\mathscr{M}_{0}$ be an arbitrary subfamily of $\mathscr{M}$ containing at most $\sigma(n-1, j)$ members, to show that $\mathscr{M}_{0}$ has an $(n-1)$-partition. The set $\mathscr{M}_{0} \cup \mathscr{L}_{0}$ consists of at most $\sigma(n-1, j)+n j+1$ members of $\mathscr{L}$. Since $n \geqslant 2$, Lemma 1 assures us that $\sigma(n-1, j)+n j+1 \leqslant \sigma(n, j)$. Hence by our hypothesis, there is an $n$-partition for $\mathscr{M}_{0} \cup \mathscr{L}_{0}$. Moreover, since $\mathscr{L}_{0}$ consists of exactly $n j+1$ members, at least $j+1$ members of $\mathscr{L}_{0}$ belong to the same partition set. Since any $j+1$ members of $\mathscr{L}_{0}$ meet only at $x, x$ must be the (unique) point of intersection for this partition set. No member of $\mathscr{M}_{0}$ contains $x$, so each member of $\mathscr{M}_{0}$ belongs to one of the remaining $n-1$ partition sets. Thus $\mathscr{M}_{0}$ has an $(n-1)$-partition. Using our induction hypothesis, it follows that $\mathscr{M}$ has an $(n-1)$ partition, and the assertion is established.

Finally, let $\mathscr{M}_{1}, \ldots, \mathscr{M}_{n-1}$ be an $(n-1)$-partition for $\mathscr{M}_{\text {. Then }} \mathscr{M}_{1}, \ldots, \mathscr{M}_{n-1}$, $\mathscr{L} \sim \mathscr{M}$ is an $n$-partition for $\mathscr{L}$, and the theorem is proved.

Facets of a $(j+1)$-dimensional simplex show that the numbers $\sigma(1, j)$ are best for each $j \geqslant 1 ; \sigma(2,1)$ is best by [4, Example 3]. When $k=3$ and $j=1$, the best bound is at least $9=\sigma(3,1)-1$, as the following example illustrates.

EXAMPle 1 . Let $\mathscr{L}$ denote the collection of 9 lines in Figure 1. Every 8 members of $\mathscr{L}$ have a 3-partition, but all 9 of them do not. Thus for $k=3$ and $j=1$, the best bound is at least 9 (and at most $\sigma(3,1)=10)$.

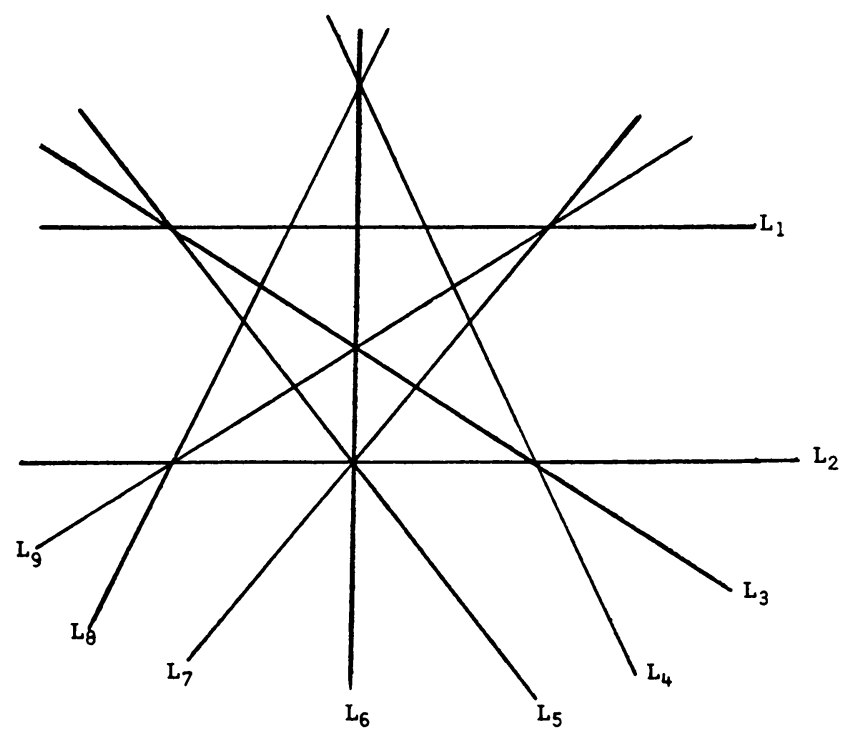

FIGURE 1 
Theorem 1 is easy to apply when $\mathscr{L}$ is a family of one-dimensional convex sets, every two of which meet in at most one point. Thus we have the following corollary.

COROLlaRY 1. If the set $S$ in $R^{d}$ has property $A_{k}$, then $S$ is a union of $k$ starshaped sets.

Proof. Let $\mathscr{L}$ be the family of all maximal convex subsets of $S$. Then $\mathscr{L}$ is a finite collection of one-dimensional convex sets, every two of which meet in at most one point. Moreover, using our hypothesis, it is easy to see that every $\sigma(k, 1)$ or fewer members of $\mathscr{L}$ have a $k$-partition. Hence by Theorem $1, \mathscr{L}$ has a $k$-partition, say $\mathscr{L}_{1}, \ldots, \mathscr{L}_{k}$. Then $\bigcup\left\{L: L\right.$ in $\left.\mathscr{L}_{i}\right\}$ is starshaped for $1 \leqslant i \leqslant k$, and $S$ is a union of $k$ starshaped sets.

Our second theorem is in the spirit of results from [1] and provides a characterization for compact unions of $k$ starshaped sets in $R^{d}$.

THEOREM 2. Let $S$ be compact in $R^{d}$. The set $S$ is a union of $k$ starshaped sets if and only if there is a sequence of compact sets $\left\{S_{i}\right\}$ converging to $S$ (relative to the Hausdorff metric) such that each $S_{i}$ has property $A_{k}$.

Proof. For the sufficiency, if each $S_{i}$ has property $A_{k}$, then by Corollary 1 each $S_{i}$ is a union of $k$ starshaped sets. Using [1, Theorem 2], $S$ is a union of $k$ starshaped sets as well.

To prove the necessity, assume that $S=T_{1} \cup \cdots \cup T_{k}$, where each $T_{m}$ is starshaped. Select $t_{m} \in \operatorname{ker} T_{m}, 1 \leqslant m \leqslant k$. For each $i \geqslant 1$, cover $S$ with finitely many open balls of radius $1 / 2 i$, and choose a point $b$ of $S$ in each ball. Define $S_{i}=\bigcup\left\{\left[b, t_{m}\right]:\left[b, t_{m}\right] \subseteq S, 1 \leqslant m \leqslant k\right\}$. Clearly each $S_{i}$ is compact and each has property $A_{k}$. Furthermore, the Hausdorff distance from $S$ to $S_{i}$ is less than $1 / i$, so the sequence $\left\{S_{i}\right\}$ converges to $S$.

\section{REFERENCES}

1. Marilyn Breen, A characterization theorem for compact unions of two starshaped sets in $R^{3}$, Pacific J. Math. 128 (1987), 63-72.

2. , A Krasnosel'skii-type theorem for unions of two starshaped sets in the plane, Pacific J. Math. 120 (1985), 19-31.

3. Clear visibility and unions of two starshaped sets in the plane, Pacific J. Math. 115 (1984), $267-275$.

4. Sets with convex closure which are a union of two starshaped sets and families of segments which have a 2-partition, J. Geom. 27 (1986), 1-23.

5. Ludwig Danzer and Branko Grünbaum, Intersection properties of boxes in $\mathbf{R}^{d}$, Combinatorica 2 (1982), 237-246.

6. Ludwig Danzer, Branko Grünbaum, and Victor Klee, Helly's theorem and its relatives, Proc. Sympos. Pure Math., vol. 7, Amer. Math. Soc., Providence, R.I., 1963, pp. 101-180.

7. M. A. Krasnosel'skii, Sur un critère pour qu'un domaine soit étoilé, Math. Sb. 19 (61) (1946), 309-310.

8. Steven R. Lay, Convex sets and their applications, Wiley, New York, 1982.

9. S. Nadler, Hyperspaces of sets, Dekker, New York, 1978.

10. F. A. Valentine, Convex sets, McGraw-Hill, New York, 1964.

Department of Mathematics, University of OKlahoma, Norman, OKlahoma 73019 